University of Nebraska - Lincoln

DigitalCommons@University of Nebraska - Lincoln

Center for Brain, Biology and Behavior: Papers \&

Publications

Brain, Biology and Behavior, Center for

$4-4-2013$

\title{
Concordance of MEG and fMRI patterns in adolescents during verb generation
}

Yingying Wang

Cincinnati Children's Hospital \& University of Cincinnati, yingying.wang@unl.edu

Scott K. Holland

Cincinnati Children's Hospital \& University of Cincinnati

Jennifer Vannest

Cincinnati Children's Hospital Research Foundation

Follow this and additional works at: https://digitalcommons.unl.edu/cbbbpapers

Part of the Behavior and Behavior Mechanisms Commons, Nervous System Commons, Other Analytical, Diagnostic and Therapeutic Techniques and Equipment Commons, Other Neuroscience and Neurobiology Commons, Other Psychiatry and Psychology Commons, Rehabilitation and Therapy Commons, and the Sports Sciences Commons

Wang, Yingying; Holland, Scott K.; and Vannest, Jennifer, "Concordance of MEG and fMRI patterns in adolescents during verb generation" (2013). Center for Brain, Biology and Behavior: Papers \& Publications. 48.

https://digitalcommons.unl.edu/cbbbpapers/48

This Article is brought to you for free and open access by the Brain, Biology and Behavior, Center for at DigitalCommons@University of Nebraska - Lincoln. It has been accepted for inclusion in Center for Brain, Biology and Behavior: Papers \& Publications by an authorized administrator of DigitalCommons@University of Nebraska - Lincoln. 


\title{
Concordance of MEG and fMRI patterns in adolescents during verb generation
}

\author{
Yingying Wang ${ }^{a, b,}$, Scott K. Holland ${ }^{a, b}$, and Jennifer Vannest ${ }^{a, c}$ \\ aPediatric Neuroimaging Research Consortium, Cincinnati Children's Hospital Research \\ Foundation, Cincinnati $\mathrm{OH}$, USA \\ bDepartment of Biomedical Engineering, University of Cincinnati, Cincinnati OH, USA \\ 'Department of Pediatrics, Division of Neurology, Cincinnati Children's Hospital Medical Center, \\ USA
}

\begin{abstract}
In this study we focused on direct comparison between the spatial distributions of activation detected by functional magnetic resonance imaging (fMRI) and localization of sources detected by magnetoencephalography (MEG) during identical language tasks. We examined the spatial concordance between MEG and fMRI results in 16 adolescents performing a three-phase verb generation task that involves repeating the auditorily presented concrete noun and generating verbs either overtly or covertly in response to the auditorily presented noun. MEG analysis was completed using a synthetic aperture magnetometry (SAM) technique, while the fMRI data were analyzed using the general linear model approach with random-effects. To quantify the agreement between the two modalities, we implemented voxel-wise concordance correlation coefficient (CCC) and identified the left inferior frontal gyrus and the bilateral motor cortex with high CCC values. At the group level, MEG and fMRI data showed spatial convergence in the left inferior frontal gyrus for covert or overt generation versus overt repetition, and the bilateral motor cortex when overt generation versus covert generation. These findings demonstrate the utility of the CCC as a quantitative measure of spatial convergence between two neuroimaging techniques.
\end{abstract}

\section{Keywords}

MEG; fMRI; Verb generation; Concordance correlation coefficient

\section{Introduction}

Human language is a complex neurocognitive process that relies upon a widely-distributed network in the brain (Bitan et al., 2005; Catani et al., 2005; Ferstl et al., 2008; Friederici et al., 2011; Karunanayaka et al., 2010; Maess et al., 2006; Price, 2010; Wilke et al., 2009). Over the past several decades, advances in modern neuroimaging techniques have offered researchers the opportunity to investigate language function in a healthy human brain noninvasively, using various techniques including positron emission tomography (PET) (Petersen et al., 1988; Wise et al., 1991), functional Magnetic Resonance Imaging (fMRI)

\footnotetext{
(C) 2012 Elsevier B.V. All rights reserved.

* Corresponding author at: Pediatric Neuroimaging Research Consortium, MLC 5033, 3333 Burnet Avenue, Cincinnati, OH 45229-3039, USA. Fax: +1 513803 1911. yingying.wang@ @chmc.org (Y. Wang)..

Appendix A. Supplementary data

Supplementary data to this article can be found online at doi:10.1016/j.brainres.2012.02.001.
} 
(Holland et al., 2001; Plante et al., 2006; Price, 2010), electroencephalography (EEG) (Pulvermuller, 1999; Spironelli and Angrilli, 2010), and magnetoencephalography (MEG) (Bowyer et al., 2004; Pylkkanen and Marantz, 2003; Salmelin, 2007; Xiang et al., 2001). PET detects increased metabolic activity using radioactive tracers and involves exposure to ionizing radiation, so it is not suitable for studying brain activity in normal, healthy children. While fMRI, as a nonionizing imaging technique, measures the blood oxygenation level dependent (BOLD) response which is an indirect measure of neuronal activity with high spatial resolution on the order of a millimeter. MEG, the magnetic counterpart of EEG, detects neuronal activity directly and provides millisecond temporal resolution. Unlike electric fields measured by EEG, which are strongly influenced by conductivity inhomogeneities within the head, the propagation of magnetic fields is not distorted by the brain, skull and scalp (Hamalainen, 1992). Therefore, localizing sources from MEG data is relatively simpler than determining the sources from EEG data.

Many studies have shown fairly good spatial convergence between MEG and fMRI responses during low-level sensory processing (auditory, visual, somatosensory) (Ahlfors et al., 1999; Auranen et al., 2009; Schulz et al., 2004; Tuunanen et al., 2003) or motor processing (Stippich et al., 1998). Fewer studies have focused on high-level cognitive functions, such as language, using both of these modalities. Previously, good spatial convergence between MEG and fMRI has been shown by tabulating individual activation regions from each modality (Lil]jestrom et al., 2009), or by computing the percentage of overlapping voxel counts within regions of interest (ROIs) (Pang et al., 2010). Divergent patterns of activation in the frontal and temporal cortex have been observed in the comparison between MEG and fMRI during a reading task with additional discrepancies between the two modalities in the left occipito-temporal cortex (Vartiainen et al., 2011). These studies hint at the importance of quantitative comparison between MEG and fMRI for mapping high-level cognitive functions to get a better understanding of brain function. Challenges remain in developing methodologies for comparing the results from MEG and fMRI quantitatively to elucidate neurophysiological processes underlying high-level cognitive functions.

In the past decade, language function has been studied extensively using fMRI. Clinical studies have shown that fMRI successfully aids specialists in determining language lateralization for epilepsy or tumor patients during presurgical evaluation (Arora et al., 2009; Dupont et al., 2010; Gaillard et al., 2002; Szaflarski et al., 2008; Yuan et al., 2006). Several published papers reviewing neuroimaging studies of language function provide a comprehensive overview of the present state of the field (Holland et al., 2007; Lee et al., 2006; Price, 2010). A typical covert verb generation task, as a paradigm for semantic association in language, produces strong left-lateralized fMRI activations. Such tasks have been used extensively in fMRI language studies of both pediatric and adult populations (Elkana et al., 2011; Frost et al., 1999; Holland et al., 2001; Szaflarski et al., 2006). The typical covert verb generation task presents a noun to the participant as a prompt to covertly generate corresponding verbs associated with the noun. For our purposes, we used a modified verb generation paradigm to provide a convenient platform for mapping the concordance between MEG and fMRI activation maps from sixteen adolescents.

In order to examine this concordance, brain activity needs to be localized in both modalities. While methods for defining active regions in fMRI are fairly well-established, there are many MEG source localization techniques. These include the minimum-norm (Hamalainen and Ilmoniemi, 1994), multi-resolution FOCUSS (Bowyer et al., 2004, 2005), beamformer (Hillebrand and Barnes, 2005), and multiple signal classification (MUSIC) (Mosher and Leahy, 1998). We chose an adaptive minimum-variance beamformer algorithm known as synthetic aperture magnetometry (SAM), for several reasons. First, SAM uses raw MEG 
data instead of an averaged dataset across multiple trials, which can capture induced brain activity that does not result in a strong average response, making it very suitable for language studies (Herdman et al., 2007; Mohamed et al., 2008; Pang et al., 2010; Wang et al., 2011; Xiang et al., 2001). Second, SAM does not need a-priori specification of the number of dipole sources (Fuchs, 2007; Vrba and Robinson, 2000). Third, SAM yields three-dimensional volumetric source maps known as statistical parametric maps (SPMs). The SPMs have been successfully compared with fMRI and have shown good spatial concurrence with the BOLD responses (Singh et al., 2002). In addition, SAM has also been successfully applied in various clinical studies (Cheyne et al., 2007; Ishii et al., 2008; Pang et al., 2010; Wang et al., 2010; Xiang et al., 2010; Zhang et al., 2011), as well as basic neuroscience research (Huo et al., 2011; Taylor et al., 2011; Wang et al., 2008). Moreover, SAM results have been suggested to be consistent with intracranial recordings of local field potentials (Gallen et al., 1995; Oshino et al., 2007).

In this study, we propose to quantify activation regions that were consistently activated in both MEG and fMRI during a verb generation task. There are a number of approaches available to quantify reproducibility measures such as voxel-based scatter plots, the Pearson correlation coefficient, the intraclass correlation coefficient, and the concordance correlation coefficient (CCC) (Lin, 1989). For continuous data, the CCC is the most popular index for assessing agreement among these methods ( $\mathrm{Li}$ and Chow, 2005). The CCC was originally developed to assess agreement between two methods on the same individual where either of the methods is treated as reference (Lawrence and Lin, 1992; Lin, 1989), and later was extended to multiple methods with or without replication (Barnhart et al., 2002, 2005; Li and Chow, 2005; Lin et al., 2002). The CCC can be expressed as the product of the Pearson correlation coefficient, which measures precision and a measure of accuracy (Lin, 1989; Williamson et al., 2007). Compared to the other available methods, the main advantages of the CCC are that it includes both precision and accuracy, is flexible, and is easy to extend to data with replication. In addition, others have already applied the CCC successfully to compare fMRI models (Lange, 1996) and reproducibility in PET images (Strother et al., 1997). The current literature has evaluated concordance between MEG and fMRI data, either by listing all the individual activation locations in a table based on suprathreshold SPMs (Liljestrom et al., 2009) or through a hypothesis-driven ROI-based analysis of overlapping voxel counts (Pang et al., 2010). In this study, we propose to use a voxel-based CCC applied to unthresholded individual SPMs to preserve objective information (Abbott et al., 2010; Strother et al., 1997).

The aim of this study is to investigate the concordance of MEG and fMRI signals corresponding to the same language paradigm performed by the same participant. Clearly, the temporal resolution of the two techniques precludes direct comparison of the time courses of brain activity corresponding to the language stimulus. Instead this study focuses on measuring the spatial concordance between MEG and fMRI to provide evidence that the two modalities are assessing the same language network during an expressive and semantic language task. Measuring spatial concordance between MEG and fMRI is an initial step establishing a framework to integrate the two modalities because it would provide confidence that fMRI could be used as a valid spatial constraint on MEG source localization using SAM or other methods.

\section{Results}

\subsection{In-scanner performance}

During the MEG session (see Table 1), the average count of verbs generated per trial for all participants was $1.8( \pm 0.5)$ and the average count of noun repetitions was $3.0( \pm 1.1)$. The average response time for overt verb generation was $2.0 \mathrm{~s}( \pm 0.3 \mathrm{~s})$, while the average 
response time for noun repetition was $1.4 \mathrm{~s}( \pm 0.2 \mathrm{~s})$. The average accuracy for overt verb generation was $95 \%( \pm 5 \%)$ and for noun repetition was $99 \%( \pm 2 \%)$. During the fMRI session (see Table 1), the average number of verbs generated per trial was $2.0( \pm 0.5)$. The average count of noun repetitions was greater with an average of $3.8( \pm 2.0)$. Verb response times among the group averaged $2.0 \mathrm{~s}( \pm 0.3 \mathrm{~s})$. Noun repetition times were more consistent, and faster, at an average of $1.4 \mathrm{~s}( \pm 0.1 \mathrm{~s})$. The average accuracy for overt verb generation was $96 \%( \pm 6 \%)$ and for noun repetition was $98 \%( \pm 5 \%)$.

\subsection{Head motion}

In MEG recordings, the head motion was measured exactly by the maximum displacement of the three coils relative to the pre-run head position. The average motion by condition was $2( \pm 1.23) \mathrm{mm}$ for covert verb generation, $2( \pm 1.27) \mathrm{mm}$ for overt verb generation and 2.07 $( \pm 1.29) \mathrm{mm}$ for overt noun repetition. For fMRI recording, the average motion by condition was estimated from the motion coregistration transformation matrix as $0.55( \pm 0.33) \mathrm{mm}$ for covert verb generation, $0.54( \pm 0.33) \mathrm{mm}$ for overt verb generation and $0.56( \pm 0.35) \mathrm{mm}$ for overt noun repetition. Analysis of variance (ANOVA) revealed that there was no significant stimulus-correlated impact on motion for both MEG and fMRI in all participants.

\subsection{Group composite activation maps}

2.3.1. C1: overt generation (say) > overt repetition (repeat)-The contrast of overt generation versus overt repetition $(\mathrm{C} 1)$ used overt repetition as a control condition to identify specific activations in the language areas for semantic processes associated with verb generation with controlling for articulation and auditory processing. The group results from MEG data for $\mathrm{C} 1$ showed a distributed left lateralized activation pattern including the pars triangular of the inferior frontal gyrus (IFG; BA 45), the pars orbitalis region of the IFG (BA 47), and the prefrontal cortex (PFC) covering superior/middle frontal gyrus (SFG/ MFG; BA 9/10), the insula (BA 13), the superior/middle temporal gyrus (STG/MTG; BA 22/42), and the inferior parietal lobule (BA 39/40) (see Fig. 2 [C1-1] and Table 2). The group results from fMRI data primarily activated the LIFG (BA 45/46/47), the PFC (BA 9/10), the medial frontal gyrus, and the cingulate gyrus (BA 32) (see Fig. 2 [C1-2] and Table 2).

2.3.2. C2: covert generation (think) > overt repetition (repeat)-The contrast of covert generation versus overt repetition (C2) also utilized overt repetition as a control condition to detect brain regions corresponding to semantic processes associated with verb generation without controlling for covert versus overt articulation. The group results from MEG data for $\mathrm{C} 2$ showed a similar left lateralized activation pattern in the BA 45 compared to $\mathrm{C} 1$, and demonstrated bilateral activation in precentral/postcentral gyrus, corresponding to sensorimotor cortex at the level of the face and mouth along the homunculus (see Fig. 2 [C2-1] and Table 2). The group results from fMRI data for $\mathrm{C} 2$ depicted significant activation in the BA 45, the most rostral portion of the IFG (BA 46), and the bilateral STG/MTG, the bilateral precentral/postcentral gyrus (see Fig. 2 [C2-2] and Table 2).

2.3.3. C3: overt generation (say) > covert generation (think)-The contrast of overt generation versus covert generation used covert generation as a control condition to isolate articulation and auditory feedback from the generation process. The group results from MEG data for C3 depicted significant activation in the left STG (BA 21/22) and postcentral gyrus (BA 40), and the right precentral gyrus (BA 6), the right insula (BA 13) (see Fig. 2 [C3-1] and Table 2). The group results from fMRI data for $\mathrm{C} 3$ also demonstrated significant activation in the BA 21/22, the bilateral BA 6, and the right BA 13 (see Fig. 2 [C3-2] and Table 2). 


\subsection{Concordance between MEG and fMRI}

Centroid of regions with $\mathrm{CCC}$ values of 0.3 and higher, reflecting moderate to nearly perfect concordance between MEG and fMRI for C1, C2 and C3, are listed in Table 3. For each region the anatomical brain area, Talairach coordinates of the centroid, maximum CCC value $\left(C C C_{m}\right)$, and number of voxels are listed. For $\mathrm{C} 1$, IFG showed moderate concordance between MEG and fMRI (see Table 3 and Fig. 3). For C2, the IFG depicted substantial concordance, and bilateral motor cortex covering precentral and postcentral gyrus showed moderate concordance between the two modalities (see Table 3 and Fig. 3). For C3, the right insula, the right precentral, and the postcentral gyrus were the regions with moderate concordance between the two modalities (see Table 3 and Fig. 3).

\section{Discussion}

In this study, we propose a voxel-wise CCC measure to quantitatively investigate the degree of spatial concordance between MEG and fMRI in the same group of adolescents performing the same verb generation task. The $\mathrm{CCC}$ has previously been used to assess the agreement between continuous measurements made by different observers (Barnhart et al., 2007; Carrasco et al., 2007; Crawford et al., 2007). It has also been successfully applied in fMRI model comparisons (Lange, 1996) and reproducibility in PET images (Strother, et al., 1997). Our CCC results indicate that high concordances between MEG and fMRI can be observed during a verb generation task. Several regions showed high concordance between the two modalities, including the left IFG, the pericentral sensorimotor regions, and the right insula. In addition, we also observed significant differences between MEG and fMRI in the left medial frontal gyrus and the left cingulate gyrus. This was not unexpected since the two modalities measure different properties of the same underlying brain activity, and similar results were previously reported (Vartiainen et al., 2011). Thus, it is very important to qualitatively and quantitatively investigate the spatial concordance between MEG and fMRI, especially during complex cognitive tasks like verb generation before the direct integration between the two.

Both overt $(\mathrm{C} 1)$ and covert $(\mathrm{C} 2)$ verb generation tasks require auditory processing, acoustic word recognition, phono-logical access and phonological working memory, semantic processing and sub-vocal word production (Karunanayaka et al., 2010). The tasks have also been found to engage visual imagery and association areas (Karunanayaka et al., 2010). In addition, overt generation engages coordination of speech articulation. Overt noun repetition engages the same sensory and cognitive modules with the exception of the semantic processing and visual imagery and association. We observed widespread cortical activations during both overt $(\mathrm{C} 1)$ and covert $(\mathrm{C} 2)$ verb generation from the group composite activation maps of MEG and fMRI. These areas covered the classical language areas including the left IFG (BA 45/46/47), left STG/MTG (BA 21/22/39/40), and SFG/MFG (BA 9/10), which were consistent with other neuroimaging studies (Binder et al., 2000; Price, 1998, 2010; Vannest et al., 2010). We hypothesize that the left IFG controls the phonological access and working memory processes needed to produce a response, following the semantic processing in the left MTG needed to construct a relevant verb response to each noun. The left STG plays a central role in acoustic word recognition, and pericentral regions are active in execution of overt responses.

Areas where MEG and fMRI group composite activation maps differed, such as the left medial frontal gyrus and the left cingulate gyrus, demonstrate the variation in regional sensitivity to brain activity between MEG and fMRI. The left medial frontal gyrus, specifically, pre-supplementary motor area (pre-SMA), has been suggested to play a role in language initiation (Crosson et al., 2001). The left cingulate gyrus has been suggested to monitor performance outcomes, response errors, and response conflicts (Botvinick et al., 
2004; Ridderinkhof et al., 2004). Why were activations in these two regions absent in MEG group composite activation maps? It could be due to the different underlying neurophysiology mechanisms between the two modalities: neuronal biomagnetic activity versus BOLD signal (Logothetis et al., 2001; Shmuel and Leopold, 2008). Some studies have shown that gamma-frequency oscillation $(30-100 \mathrm{~Hz})$ was associated with SMA activations (Brovelli et al., 2005; Jensen et al., 2007). We only included 1-30 Hz in SAM analysis, which could also contribute to the discrepancies between the two modalities. As expected, significantly greater activation was identified in the bilateral primary motor cortex during the overt versus covert verb generation (C3) for both MEG and fMRI group results, which is consistent with our previous publication on this task (Vannest et al., 2010) and other investigators (Huang et al., 2002; Soros et al., 2006). An alternative explanation for the differences between fMRI and MEG composite activation maps in Fig. 2 stems from the difference in the way in which source location is computed in the two modalities. In fMRI the location of the BOLD signal is provided by the Fourier transformation of the raw MRI signal and its differences between conditions. With MEG the source of brain activity is localized using SAM which is particularly insensitive to centralized sources where the phase differences among sensor signals are minimal. The fact that the differences between the composite activation maps occur near mid-line areas of the brain could therefore reveal one of the weaknesses of MEG source localization and at the same time emphasize the strength of the method we are proposing to spatially localize sources based on the fMRI data.

The CCC is a convenient quantitative index for evaluating reproducibility of a continuous variable to assess whether different methods produce similar (reproducible) values (Crawford et al., 2007; Williamson et al., 2007). The CCC has been shown to be identical to a specific intraclass correlation coefficient (ICC) (Mcgraw and Wong, 1996; Shrout and Fleiss, 1979) using the variance components from the linear mixed model in which observers are considered as a fixed effect and subjects as a random effect (Carrasco and Jover, 2003). However, the expression of CCC is easier to understand and implement than ICC (Carrasco and Jover, 2003). In this study, we implemented voxel-wise CCC to quantitatively measure the concordance between MEG and fMRI. For C1, the left IFG showed moderate concordance $(\mathrm{CCC}=0.48$, see Table 3 ) between MEG and fMRI, revealing the consistent role of phonological processing in the generation of a semantically related verb. For $\mathrm{C} 2$, the left IFG showed even greater concordance between modalities $(\mathrm{CCC}=0.78$, see Table 3), while the bilateral pre- and postcentral gyri showed moderate concordances between MEG and fMRI data. For C3, bilateral pre- and postcentral gyri, and right insula had moderate concordances between the two modalities $(\mathrm{CCC}=0.39,0.37,0.45$ respectively), emphasizing the consistent role of speech motor circuitry residing in the contrast between the overt and covert responses.

Motivation for this analysis of the concordance between MEG and fMRI during the same language task is that we are interested in modeling the connectivity and information flow within the neural circuitry supporting language functions in the human brain. The main focus of this current study is to provide the converging evidence by using CCC to measure the concordance between MEG and fMRI in order to ensure that the two modalities are assessing the same language network. To achieve this goal, we used a broad bandpass (0.6$30 \mathrm{~Hz}$ ) and a long time window ( $3 \mathrm{~s})$ in order to capture most brain activity and present a "big picture" of the verb generation process, instead of using different frequency bands and time windows. In addition, CCC can be utilized to assess the concordance between the two modalities in different time windows and frequency bands (delta, alpha, theta, gamma, etc.). However, it is out of the goal and scope of this present study. Our future work will fully utilize the high spatial resolution of fMRI data to extract time series with high temporal information from MEG data from all regions relevant to the language task, which will improve estimates of connectivity within the language network, and provide better 
assessments of changes that occur in the language networks during brain development. (Karunanayaka et al., 2011).

The direct comparison between MEG and fMRI data from sixteen adolescents during a verb generation task using CCC demonstrates the spatial convergence in several brain areas involving classic language regions and primary motor cortex. While the relationship between BOLD-effects and electrophysiological responses for mapping complex cognitive processes such as language is still incomplete, the spatial overlap between MEG and fMRI from our study suggests that the two modalities assess the same neural substrate in several aspects, but in different ways and with different advantages and disadvantages. Convolution of neural activation with the hemodynamic response function of the cerebrovascular system to produce the BOLD signal observed by fMRI, together with relatively slow sampling rate do not permit fMRI to monitor brain function on a neurophysiologic time-scale. MEG, on the other hand, offers a high temporal resolution but with some limitations on the precision of source localization. Thus, combining the two can potentially capitalize on the advantages of each modality and obviate the primary limitations of each, leading to an improved method for brain network mapping and connectivity analysis in the future. Optimal integration methods of MEG and fMRI must address the discrepancies between the two modalities. Meanwhile, the high temporal resolution of MEG source waveforms with fMRI guided spatial resolution can lead to better understanding of the temporal dynamics of the neural networks supporting the language processing. A potential clinical benefit of multimodal integration as demonstrated here between MEG and fMRI include applications for seizure localization and surgical planning for epilepsy.

\section{Experimental procedures}

\subsection{Participants}

Nineteen healthy, native-English-speaking adolescents were drawn from a longitudinal subgroup recruited from a larger cross-sectional sample of participants previously included in our fMRI studies of language development (Holland et al., 2007) (NIH grant R01HD38578). Informed consent or assent was given by all parents and participants. This study was approved by the Institutional Review Board at Cincinnati Children's Hospital Medical Center. None of the participants had any neurological impairment or neurological trauma. Data from three participants were excluded due to the extensive noise in the MEG data which was caused by permanent dental retainers. MEG and fMRI data from sixteen participants were included in this study with average age 15.8 year $\pm 1.2($ mean \pm SD, Table 1). The handedness was assessed according to the Edinburgh test (Oldfield, 1971).

\subsection{Paradigm}

We designed an event-related fMRI acquisition with auditory stimuli presented during completely silent gradient intervals, which also allows us to record the participants' performance when they articulate the verbs (Schmithorst and Holland, 2004). This method fully utilized the hemodynamic delay of the BOLD response and collected the image data at the peak of the response post-stimuli. Thus, using the event-related design we were able to determine the correlations between the participants' performance with the fMRI activations in the classical language areas (Vannest et al., 2010). Another advantage is that it minimizes the motion artifacts which might be induced by the articulation since the actual data acquisition happens after the articulation processing. Finally, event-related study design also allows us to implement the same paradigm from fMRI to the corresponding MEG study. So we can compare the brain responses from both techniques during the same covert and overt verb generation task. 
The adolescent participants listened to concrete nouns with visual instructions and responded with related verbs covertly and overtly during both MEG and fMRI sessions. All the concrete nouns were selected from the MRC Psycholinguistic Database (Coltheart, 1981 ), and controlled by the number of syllables (range $1-2$, mean \pm SD: $1.33 \pm 0.47$ ), as well as length (range 3-9, mean \pm SD: $4.77 \pm 1.19$ ). And the difficulty of the words was assessed by the age of acquisition rating (range 150-300, mean \pm SD: $245.60 \pm 37.97$ ). The words were binaurally presented at $70 \mathrm{~dB}$. For example, if the participant hears the noun "apple", he/she might generate "eat" or "bite". Participants were visually prompted to generate verbs either overtly or covertly in an interleaved fashion. As a control task, the participants were prompted to overtly repeat the noun. The stimuli and timing of presentations for the MEG and fMRI paradigms were identical, though more trials were used with MEG to enhance the signal to noise ratio. MEG stimuli included three conditions: covert verb generation (think), overt verb generation (say), and overt noun repetition (repeat), which were presented with BrainX software (Xiang et al., 2001). The MEG paradigm included 30 5-s trials for each condition and 90 5-s trials in total (see [M1] in Fig. 1). FMRI stimuli were presented using DirectRT software (Empirisoft Corp., NYC, NY). The fMRI paradigm included 15 cycles of 36-s, each consisting of 3 12-s trials: covert verb generation (think), overt verb generation (say), and overt noun repetition (repeat) (see [F1] in Fig. 1).

\subsection{Data acquisition}

We used a 275-channel whole head MEG system (VSM Med-Tech Ltd., Port Coquitlam, BC, Canada) to acquire all the MEG data in a magnetically shielded room (MSR) (VacuumSchmelze, Hanau, Germany). For each participant, the head position with respect to the sensor array was determined by three coils placed on the nasion and at the left and right preauricular points. Digital photos of the three coils were also taken to assist with the placement of three fiducial points on the participant before the MRI/fMRI scan. The three fiducial points established the coordinate transformation between MEG and fMRI. The MEG data were filtered offline by a $150 \mathrm{~Hz}$ low-pass filter and sampled at $6 \mathrm{KHz}$. All the participants were instructed to avoid eye blinks and head movements during the recording procedure. We used continuous head localization method to monitor the head movements during the MEG acquisition and to eliminate the bad data segments and determine the stimulus-related motions.

FMRI data were acquired on a Philips Achieva 3-T MRI scanner with Dual Quasar gradients (Philips Medical Systems, Best, The Netherlands). Before the fMRI acquisition, three fiducial markers were placed in identical locations to the ones used in the MEG recordings so that MEG results could be co-registered precisely to the anatomical MRI. A T2*weighted, gradient-echo, sparse acquisition, echo planar imaging (EPI) sequence was used with parameters: TR/TE $=2000 / 38 \mathrm{~ms}, \mathrm{FOV}=24 \times 24 \mathrm{~cm}$, matrix $=64 \times 64$, slice thickness $=5$ $\mathrm{mm}$. Thirty-five slices were acquired at 137 time points for a total imaging time of $9 \mathrm{~min}$. The initial 2 time points were discarded to allow the protons to reach $\mathrm{T} 1$ relaxation equilibrium. In addition, a high-resolution T1-weighted 3D anatomical scan was acquired using an inversion recovery (IR) prepared turbo gradient echo acquisition protocol with parameters: TR/TI/TE $=8.1 / 1052 / 3.7 \mathrm{~ms}, \mathrm{FOV}=25 \times 25 \times 18 \mathrm{~cm}$, spatial resolution of $1 \times 1 \times 1$ $\mathrm{mm}$. Audiovisual stimuli were presented using an MRI compatible audio-video system with binocular goggles and pneumatic headphones (Avotec, Inc. SS3100/SV7021). Verbal responses were recorded via in-scanner microphone included with this system. Among all the participants, six of them had fMRI scan before MEG scan, and ten of them had MEG scan before fMRI scan. The average interval between the two scans was 15 days. 


\subsection{Data processing}

Based on the routine noise test before the participant entered the MEG room, noisy channels in the MEG data were identified by visual inspection of the frequency plot and excluded

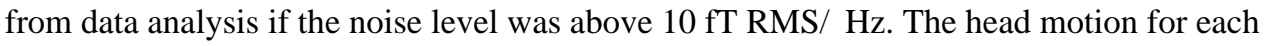
participant was computed in MATLAB (The MathWorks, Inc., Natick, MA) at each time point using the continuous head localization channels (HLC11-13, 18, 21-23, 28, 31-33, 38). Stimulus-correlated motion was evaluated by analyzing the time series of displacements. For each participant, the mean motion in all directions for each trial and each stimulus was analyzed for the main effect of stimulus using analysis of variance (ANOVA). We classified a sample as "bad" if the root mean square (RMS) of all three displacements in $\mathrm{x}, \mathrm{y}, \mathrm{z}$ directions exceeded $6 \mathrm{~mm}$ for any fiducial markers (nasion, left or right pre-auricular makers). $6 \mathrm{~mm}$ is the median of motion in all participants during MEG scan, which is also within the acceptable range of head movements suggested by literature (Herdman and Cheyne, 2009). Then, a trial was rejected if more than $10 \%$ of the total samples in the trial were "bad". In addition, a trial was rejected if the participant did not respond at all. After removal of the bad trials, unaveraged MEG data were analyzed using SAM with a window length of 3-s, which allowed us to capture all the brain activity after auditory stimuli onset.

MEG waveforms were downsampled from $6 \mathrm{KHz}$ to $600 \mathrm{~Hz}$ and bandpass filtered at 0.6-30 $\mathrm{Hz}$. The task frequency was about $0.2 \mathrm{~Hz}$, and the Fourier transform of all the MEG sensor data showed $95 \%$ power within the frequency range of $0.6-30 \mathrm{~Hz}$. Thus, a frequency band from $0.6-30 \mathrm{~Hz}$ was used to generate SAM images of three contrasts including overt versus covert, covert versus repetition, and overt versus repetition using the Student $t$-test. For group analysis, the individual $S P M_{\{t\}}$ was transformed into Talairach space (Lancaster et al., 2000) by applying the transformation matrix derived from the anatomical MRI to Talairach space using 3dWarp in AFNI (analysis and visualization of functional magnetic resonance image) (Cox, 1996). MEG SAM results were also aligned with the anatomical MRI by the three fiducial marks. Then, the transformation matrix, derived from the anatomical MRI transformed into Talairach space, was also applied to MEG SAM maps in AFNI. Thus, both modalities were transformed into Talairach space using the same transformation matrix derived from the anatomical MRI to Talairach space. Then, we performed a one-sample $t$ test on the individual Talairach-transformed SAM results on a voxel by voxel basis to determine which brain regions showed significant activation for the three contrasts, respectively. To control for multiple comparisons, statistically significant effects were tested with false discovery rate (FDR) (Genovese et al., 2002). The threshold was set at p<.05, corrected by FDR.

The fMRI data analysis was performed using routines written in IDL (ITT Visual Information Solutions, Boulder, CO) and Cincinnati Children's Hospital Image Processing Software. EPI data were corrected for Nyquist ghosts and geometric distortion using a multiecho reference method (Schmithorst et al., 2001), retrospectively motion-corrected using pyramid co-registration (Yuan et al., 2009), and then spatially normalized into Talairach space using a linear affine transformation (Wilke et al., 2002). The transformation matrix was saved for transforming MEG SAM results to Talairach space. The square root of the sum of the square of all six parameters (translation parameters in $\mathrm{x}, \mathrm{y}, \mathrm{z}$, and rotation parameters) was analyzed for the main effect of stimulus using ANOVA. We used a general linear model and random-effects analysis to generate significant group activations in three contrasts including overt generation versus covert repetition, covert generation versus overt generation, and overt generation versus covert generation (Vannest et al., 2010). The threshold for group activation maps was set at $\mathrm{p}<0.05$, corrected by Monte Carlo simulation (Schmithorst and Holland, 2006). 
Because the spatial resolution of fMRI and MEG differ, some interpolation is required in order to resample the data to the same resolution prior to computing the CCC maps. The fMRI data was acquired at $4 * 4 * 5 \mathrm{~mm}$, while the spatial resolution of MEG data was determined by a three-dimensional grid of possible source locations for SAM analysis. There is a trade-off between the ability to resolve sources that are close together and the possibility of introducing spurious sources. With widely spaced grid points (e.g., $10 * 10 * 10$ $\mathrm{mm}$ ) the likelihood of observing spurious beamformer activities is relatively low (Green and Mcdonald, 2009). Taking this into consideration, we used $7 * 7 * 7 \mathrm{~mm}$ for our SAM analysis and generated 13,041 voxels over the entire volume of the brain. Using a finer grid to match the spatial resolution of fMRI data $(4 * 4 * 5 \mathrm{~mm})$ would likely increase the spurious sources. Therefore, we interpolated the fMRI data to match the MEG resolution at $7 * 7 * 7$ resolution prior to computing the CCC. A recent study by Dr. Pang set a precedent for this approach by resampling the functional volumes obtained using finer grids with fMRI to match the coarser grid of MEG (Pang et al., 2010).

\subsection{Comparison between MEG and fMRI}

For the concordance measures, the fMRI SPM $\{t\}$ maps were exported from in-house IDL software package to AFNI and were re-sampled to match the MEG SAM image grids. Then, the $\mathrm{SPM}_{\{\mathrm{t}\}}$ from both MEG and fMRI were converted to $\mathrm{SPM}_{\{\mathrm{z}\}}$. We utilized the CCC macro written in R (Crawford et al., 2007) and developed our own bash script that provided a voxel-wise CCC package working with AFNI, which can be downloaded from the following website: http://homepages.uc.edu/ wang2yg/yw_3dccc_v0605.zip or directly contact the authors. The CCC was calculated using the following formulas (Crawford et al.,

2007): $C C C=\frac{2 \sigma_{12}}{\sigma_{1}^{2}+\sigma_{2}{ }^{2}+\left(\mu_{1}-\mu_{2}\right)^{2}}$, where $\mu_{1}$ and $\mu_{2}$ are the means for the two variables (for our study, z-score values of SPMs from MEG and fMRI), $\sigma_{1}^{2}$ and $\sigma_{2}^{2}$ are the corresponding variances, and $\sigma_{12}$ is the covariance. In this study, for a given voxel, the CCC was computed on an $\mathrm{N}$-length data set $(\mathrm{N}=16)$ by the following formulas:

$$
\begin{aligned}
\mathrm{CCC} & =1-\frac{E\left[\left(\mathrm{fMRI}_{j}-\mathrm{MEG}_{j}\right)^{2}\right]}{E\left[\left(\mathrm{fMRI}_{j}-\mathrm{MEG}_{j}\right)^{2} \mid \mathrm{fMRI}_{j}, \mathrm{MEG}_{j} \quad \text { are independent }\right]} \\
& =\frac{2 \sigma_{\mathrm{fMRI}_{j}} \sigma_{\mathrm{MEG}_{j}} \rho}{\sigma_{\mathrm{fMRI}_{j}}^{2}+\sigma_{\mathrm{MEG}_{j}}^{2}+\left(\mu_{\mathrm{fMRI}_{j}}-\mu_{\mathrm{MEG}_{j}}\right)^{2}}=\rho \chi_{a},
\end{aligned}
$$

where $\rho=\operatorname{Corr}\left(\mathrm{fMRI}_{j}, \mathrm{MEG}_{j}\right)$ is referred to as the precision component, equivalent to the

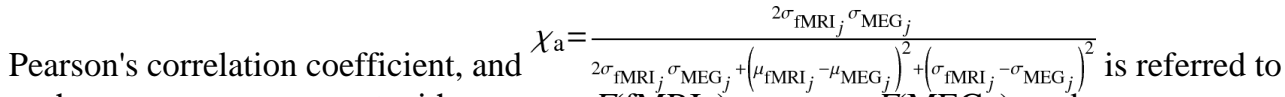
as the accuracy component with $\mu_{\mathrm{fMRIj}}=E\left(\mathrm{fMRI}_{i j}\right), \mu_{\mathrm{MEG}_{j}}=E\left(\mathrm{MEG}_{i j}\right)$, and $\sigma_{\mathrm{fMRI}_{j}}^{2}=\operatorname{Var}\left(\mathrm{fMRI}_{i j}\right), \sigma_{\mathrm{MEG}_{j}}^{2}=\operatorname{Var}\left(\mathrm{MEG}_{i j}\right)$. We note that $\chi_{a}$ assesses location shift $\left(\left(\mu_{\mathrm{fMRI}_{j}-}\right.\right.$ $\left.\left.\mu_{\mathrm{MEG}_{j}}\right)^{2}\right)$ and scale shift $\left(\left(\sigma_{\mathrm{fMRI}_{j}}-\sigma_{\mathrm{MEG}_{j}}\right)^{2}\right)$ relative to the scales $\left(\sigma_{\mathrm{fMRI}_{j}}, \sigma_{\mathrm{MEG}_{j}}\right)$. For fixed values of location and scale shifts, $\chi_{a}$ is a function of $\left(\sigma_{\mathrm{fMRI}_{j}}, \sigma_{\mathrm{MEG}_{j}}\right)$ and when $\chi_{a} \rightarrow 1$, $\mathrm{CCC} \rightarrow \rho$. Thus, $\mathrm{CCC}$ measures the real concordance rather than merely correlation (CCC $\$ \rho$ ). The CCC has the scale of 1 (perfect agreement) to 0 (no agreement) to -1 (perfect reversed agreement). We aimed to find the regions with high agreement between the two modalities so only the positive CCC values were counted in this study. Then, the CCC maps were spatially filtered using a $6 \mathrm{~mm}$ Gaussian filter, thresholded at 0.3 , and limited to clusters of size greater than or equal to 10 contiguous voxels to correct for the occurrence of spurious individual voxels and improve the visualization of the CCC maps in Fig. 3. This threshold was selected based upon benchmarks used for categorizing the concordance 
results: $0.3-0.5$ (moderate concordance), $0.51700-0.8$ (substantial concordance), $0.81-1$ (almost perfect concordance) (Eaton et al., 2008).

\section{Supplementary Material}

Refer to Web version on PubMed Central for supplementary material.

\section{Acknowledgments}

This study was supported by a grant from the U.S. National Institute of Health (NIH grant R01-HD38578, P.I. SK Holland). The authors acknowledge the assistance of Ms. Amanda Huber, Ms. Sara Robertson and Ms. Julie Franks, for helping with recruitments and data collection, and Mr. Kendall O'Brien and Ms. Amanda Woods, for performing all the MRI scans.

\section{REFERENCES}

Abbott DF, Waites AB, Lillywhite LM, Jackson GD. fMRI assessment of language lateralization: an objective approach. NeuroImage. 2010; 50:1446-1455. [PubMed: 20097290]

Ahlfors SP, Simpson GV, Dale AM, Belliveau JW, Liu AK, Korvenoja A, Virtanen J, Huotilainen M, Tootell RB, Aronen HJ, Ilmoniemi RJ. Spatiotemporal activity of a cortical network for processing visual motion revealed by MEG and fMRI. J. Neurophysiol. 1999; 82:2545-2555. [PubMed: 10561425]

Arora J, Pugh K, Westerveld M, Spencer S, Spencer DD, Todd Constable R. Language lateralization in epilepsy patients: fMRI validated with the Wada procedure. Epilepsia. 2009; 50:2225-2241. [PubMed: 19490042]

Auranen T, Nummenmaa A, Vanni S, Vehtari A, Hamalainen MS, Lampinen J, Jaaskelainen IP. Automatic fMRI-guided MEG multidipole localization for visual responses. Hum. Brain Mapp. 2009; 30:1087-1099. [PubMed: 18465749]

Barnhart HX, Haber M, Song J. Overall concordance correlation coefficient for evaluating agreement among multiple observers. Biometrics. 2002; 58:1020-1027. [PubMed: 12495158]

Barnhart HX, Song J, Haber MJ. Assessing intra, inter and total agreement with replicated readings. Stat. Med. 2005; 24:1371-1384. [PubMed: 15570569]

Barnhart HX, Haber MJ, Lin LI. An overview on assessing agreement with continuous measurements. J. Biopharm. Stat. 2007; 17:529-569. [PubMed: 17613641]

Binder J, Frost J, Hammeke T, Bellgowan P, Springer J, Kaufman J, Possing E. Human temporal lobe activation by speech and nonspeech sounds. Cereb. Cortex. 2000; 10:512. [PubMed: 10847601]

Bitan T, Booth JR, Choy J, Burman DD, Gitelman DR, Mesulam MM. Shifts of effective connectivity within a language network during rhyming and spelling. J. Neurosci. 2005; 25:5397-5403. [PubMed: 15930389]

Botvinick MM, Cohen JD, Carter CS. Conflict monitoring and anterior cingulate cortex: an update. Trends Cogn. Sci. 2004; 8:539-546. [PubMed: 15556023]

Bowyer SM, Moran JE, Mason KM, Constantinou JE, Smith BJ, Barkley GL, Tepley N. MEG localization of language-specific cortex utilizing MR-FOCUSS. Neurology. 2004; 62:2247-2255. [PubMed: 15210890]

Bowyer SM, Moran JE, Weiland BJ, Mason KM, Greenwald ML, Smith BJ, Barkley GL, Tepley N. Language laterality determined by MEG mapping with MR-FOCUSS. Epilepsy Behav. 2005; 6:235-241. [PubMed: 15710310]

Brovelli A, Lachaux JP, Kahane P, Boussaoud D. High gamma frequency oscillatory activity dissociates attention from intention in the human premotor cortex. NeuroImage. 2005; 28:154 164. [PubMed: 16023374]

Carrasco JL, Jover L. Estimating the generalized concordance correlation coefficient through variance components. Biometrics. 2003; 59:849-858. [PubMed: 14969463]

Carrasco JL, Jover L, King TS, Chinchilli VM. Comparison of concordance correlation coefficient estimating approaches with skewed data. J. Biopharm. Stat. 2007; 17:673-684. [PubMed: 17613647] 
Catani M, Jones DK, Ffytche DH. Perisylvian language networks of the human brain. Ann. Neurol. 2005; 57:8-16. [PubMed: 15597383]

Cheyne D, Bostan AC, Gaetz W, Pang EW. Event-related beamforming: a robust method for presurgical functional mapping using MEG. Clin. Neurophysiol. 2007; 118:1691-1704. [PubMed: 17587643]

Coltheart M. The MRC psycholinguistic database. Q. J. Exp. Psychol. 1981; 33:497-505.

Cox RW. AFNI: software for analysis and visualization of functional magnetic resonance neuroimages. Comput. Biomed. Res. 1996; 29:162-173. [PubMed: 8812068]

Crawford SB, Kosinski AS, Lin HM, Williamson JM, Barnhart HX. Computer programs for the concordance correlation coefficient. Comput. Meth. Programs Biomed. 2007; 88:62-74.

Crosson B, Sadek JR, Maron L, Gokcay D, Mohr CM, Auerbach EJ, Freeman AJ, Leonard CM, Briggs RW. Relative shift in activity from medial to lateral frontal cortex during internally versus externally guided word generation. J. Cogn. Neurosci. 2001; 13:272-283. [PubMed: 11244551]

Dupont S, Duron E, Samson S, Denos M, Volle E, Delmaire C, Navarro V, Chiras J, Lehéricy S, Samson Y. Functional MR Imaging or Wada Test: which is the better predictor of individual postoperative memory outcome? Radiology. 2010; 255:128. [PubMed: 20308450]

Eaton KP, Szaflarski JP, Altaye M, Ball AL, Kissela BM, Banks C, Holland SK. Reliability of fMRI for studies of language in post-stroke aphasia subjects. NeuroImage. 2008; 41:311-322. [PubMed: 18411061]

Elkana O, Frost R, Kramer U, Ben-Bashat D, Schweiger A. Cerebral language reorganization in the chronic stage of recovery: a longitudinal fMRI study. Cortex. 2011 (Sep 14, Electronic publication ahead of print).

Ferstl EC, Neumann J, Bogler C, Von Cramon DY. The extended language network: a meta-analysis of neuroimaging studies on text comprehension. Hum. Brain Mapp. 2008; 29:581-593. [PubMed: 17557297]

Friederici AD, Brauer J, Lohmann G. Maturation of the language network: from inter- to intrahemispheric connectivities. PLoS One. 2011; 6:e20726. [PubMed: 21695183]

Frost JA, Binder JR, Springer JA, Hammeke TA, Bellgowan PSF, Rao SM, Cox RW. Language processing is strongly left lateralized in both sexes. Brain. 1999; 122:199-208. [PubMed: 10071049]

Fuchs, A. Beamforming and its applications to brain connectivity.. In: Jirsa, VK.; Mcintosh, AR., editors. Handbook of Brain Connectivity. Springer Verlag; Berlin: 2007. p. 357-378.

Gaillard WD, Balsamo L, Xu B, Grandin CB, Braniecki SH, Papero PH, Weinstein S, Conry J, Pearl PL, Sachs B, Sato S, Jabbari B, Vezina LG, Frattali C, Theodore WH. Language dominance in partial epilepsy patients identified with an fMRI reading task. Neurology. 2002; 59:256-265. [PubMed: 12136067]

Gallen CC, Schwartz BJ, Bucholz RD, Malik G, Barkley GL, Smith J, Tung H, Copeland B, Bruno L, Assam S, Hirschkoff E, Bloom F. Presurgical localization of functional cortex using magnetic source imaging. J. Neurosurg. 1995; 82:988-994. [PubMed: 7760203]

Genovese CR, Lazar NA, Nichols T. Thresholding of statistical maps in functional neuroimaging using the false discovery rate. NeuroImage. 2002; 15:870-878. [PubMed: 11906227]

Green, JJ.; Mcdonald, JJ. A practical guide to beamformer source reconstruction for EEG.. In: Hansen, PC.; Kringelbach, ML.; Salmelin, R., editors. Brain Signal Analysis: Advances in Neuroelectric and Neuromagnetic Methods. The MIT Press; Massachusetts: 2009. p. 76-98.

Hamalainen MS. Magnetoencephalography: a tool for functional brain imaging. Brain Topogr. 1992; 5:95-102. [PubMed: 1489655]

Hamalainen MS, Ilmoniemi RJ. Interpreting magnetic fields of the brain: minimum norm estimates. Med. Biol. Eng. Comput. 1994; 32:35-42. [PubMed: 8182960]

Herdman, AT.; Cheyne, D. A practical guide for MEG and beamforming.. In: Hansen, PC.; Kringelbach, ML.; Salmelin, R., editors. Brain Signal Analysis: Advances in Neuroelectric and Neuromagnetic Methods. The MIT Press; Massachusetts: 2009. p. 105-106.

Herdman AT, Pang EW, Ressel V, Gaetz W, Cheyne D. Task-related modulation of early cortical responses during language production: an event-related synthetic aperture magnetometry study. Cereb. Cortex. 2007; 17:2536-2543. [PubMed: 17204818] 
Hillebrand A, Barnes GR. Beamformer analysis of MEG data. Int. Rev. Neurobiol. 2005; 68:149-171. [PubMed: 16443013]

Holland SK, Plante E, Weber Byars A, Strawsburg RH, Schmithorst VJ, Ball WS Jr. Normal fMRI brain activation patterns in children performing a verb generation task. NeuroImage. 2001; 14:837-843. [PubMed: 11554802]

Holland SK, Vannest J, Mecoli M, Jacola LM, Tillema JM, Karunanayaka PR, Schmithorst VJ, Yuan W, Plante E, Byars AW. Functional MRI of language lateralization during development in children. Int. J. Audiol. 2007; 46:533-551. [PubMed: 17828669]

Huang J, Carr TH, Cao Y. Comparing cortical activations for silent and overt speech using eventrelated fMRI. Hum. Brain Mapp. 2002; 15:39-53. [PubMed: 11747099]

Huo X, Wang Y, Kotecha R, Kirtman EG, Fujiwara H, Hemasilpin N, Degrauw T, Rose DF, Xiang J. High gamma oscillations of sensorimotor cortex during unilateral movement in the developing brain: a MEG study. Brain Topogr. 2011; 23:375-384. [PubMed: 20577795]

Ishii R, Canuet L, Ochi A, Xiang J, Imai K, Chan D, Iwase M, Takeda M, Snead OC III, Otsubo H. Spatially filtered magnetoencephalography compared with electrocorticography to identify intrinsically epileptogenic focal cortical dysplasia. Epilepsy Res. 2008; 81:228-232. [PubMed: 18672350]

Jensen O, Kaiser J, Lachaux JP. Human gamma-frequency oscillations associated with attention and memory. Trends Neurosci. 2007; 30:317-324. [PubMed: 17499860]

Karunanayaka P, Schmithorst VJ, Vannest J, Szaflarski JP, Plante E, Holland SK. A group independent component analysis of covert verb generation in children: a functional magnetic resonance imaging study. NeuroImage. 2010; 51:472-487. [PubMed: 20056150]

Karunanayaka P, Schmithorst VJ, Vannest J, Szaflarski JP, Plante E, Holland SK. A linear structural equation model for covert verb generation based on independent component analysis of fMRI data from children and adolescents. Front. Syst. Neurosci. 2011; 5:29. [PubMed: 21660108]

Lancaster JL, Woldorff MG, Parsons LM, Liotti M, Freitas CS, Rainey L, Kochunov PV, Nickerson D, Mikiten SA, Fox PT. Automated Talairach atlas labels for functional brain mapping. Hum. Brain Mapp. 2000; 10:120-131. [PubMed: 10912591]

Lange N. Statistical approaches to human brain mapping by functional magnetic resonance imaging. Stat. Med. 1996; 15:389-428. [PubMed: 8668868]

Lawrence I, Lin K. Assay validation using the concordance correlation coefficient. Biometrics. 1992:599-604.

Lee A, Kannan V, Hillis AE. The contribution of neuroimaging to the study of language and aphasia. Neuropsychol. Rev. 2006; 16:171-183. [PubMed: 17160697]

Li R, Chow M. Evaluation of reproducibility for paired functional data. J. Multivar. Anal. 2005; 93:81-101. [PubMed: 19458785]

Liljestrom M, Hulten A, Parkkonen L, Salmelin R. Comparing MEG and fMRI views to naming actions and objects. Hum. Brain Mapp. 2009; 30:1845-1856. [PubMed: 19378277]

Lin LI. A concordance correlation coefficient to evaluate reproducibility. Biometrics. 1989; 45:255. [PubMed: 2720055]

Lin L, Hedayat A, Sinha B, Yang M. Statistical methods in assessing agreement. J. Am. Stat. Assoc. 2002; 97:257-270.

Logothetis NK, Pauls J, Augath M, Trinath T, Oeltermann A. Neurophysiological investigation of the basis of the fMRI signal. Nature. 2001; 412:150-157. [PubMed: 11449264]

Maess B, Herrmann CS, Hahne A, Nakamura A, Friederici AD. Localizing the distributed language network responsible for the N400 measured by MEG during auditory sentence processing. Brain Res. 2006; 1096:163-172. [PubMed: 16769041]

Mcgraw KO, Wong S. Forming inferences about some intraclass correlation coefficients. Psychol. Meth. 1996; 1:30.

Mohamed IS, Cheyne D, Gaetz WC, Otsubo H, Logan WJ, Carter Snead O III, Pang EW. Spatiotemporal patterns of oscillatory brain activity during auditory word recognition in children: a synthetic aperture magnetometry study. Int. J. Psychophysiol. 2008; 68:141-148. [PubMed: 18359115] 
Mosher JC, Leahy RM. Recursive MUSIC: a framework for EEG and MEG source localization. IEEE Trans. Biomed. Eng. 1998; 45:1342-1354. [PubMed: 9805833]

Oldfield RC. The assessment and analysis of handedness: the Edinburgh inventory. Neuropsychologia. 1971; 9:97-113. [PubMed: 5146491]

Oshino S, Kato A, Wakayama A, Taniguchi M, Hirata M, Yoshimine T. Magnetoencephalographic analysis of cortical oscillatory activity in patients with brain tumors: synthetic aperture magnetometry (SAM) functional imaging of delta band activity. NeuroImage. 2007; 34:957-964. [PubMed: 17175174]

Pang EW, Wang F, Malone M, Kadis DS, Donner EJ. Localization of Broca's area using verb generation tasks in the MEG: validation against fMRI. Neurosci. Lett. 2010; 490:215-219. [PubMed: 21195135]

Petersen SE, Fox PT, Posner MI, Mintun M, Raichle ME. Positron emission tomographic studies of the cortical anatomy of single-word processing. Nature. 1988; 331:585-589. [PubMed: 3277066]

Plante E, Schmithorst VJ, Holland SK, Byars AW. Sex differences in the activation of language cortex during childhood. Neuropsychologia. 2006; 44:1210-1221. [PubMed: 16303148]

Price CJ. The functional anatomy of word comprehension and productioin. Trends Cogn. Sci. 1998; 8:281-288. [PubMed: 21227210]

Price CJ. The anatomy of language: a review of 100 fMRI studies published in 2009. Ann. NY Acad. Sci. 2010; 1191:62-88. [PubMed: 20392276]

Pulvermuller F. Words in the brain's language. Behav. Brain Sci. 1999; 22:253-336. [PubMed: 11301524]

Pylkkanen L, Marantz A. Tracking the time course of word recognition with MEG. Trends Cogn. Sci. 2003; 7:187-189. [PubMed: 12757816]

Ridderinkhof KR, Ullsperger M, Crone EA, Nieuwenhuis S. The role of the medial frontal cortex in cognitive control. Science. 2004; 306:443. [PubMed: 15486290]

Salmelin R. Clinical neurophysiology of language: the MEG approach. Clin. Neurophysiol. 2007; 118:237-254. [PubMed: 17008126]

Schmithorst VJ, Holland SK. Event-related fMRI technique for auditory processing with hemodynamics unrelated to acoustic gradient noise. Magn. Reson. Med. 2004; 51:399-402. [PubMed: 14755667]

Schmithorst VJ, Holland SK. Functional MRI evidence for disparate developmental processes underlying intelligence in boys and girls. NeuroImage. 2006; 31:1366-1379. [PubMed: 16540350]

Schmithorst VJ, Dardzinski BJ, Holland SK. Simultaneous correction of ghost and geometric distortion artifacts in EPI using a multiecho reference scan. IEEE Trans. Med. Imaging. 2001; 20:535-539. [PubMed: 11437113]

Schulz M, Chau W, Graham SJ, Mcintosh AR, Ross B, Ishii R, Pantev C. An integrative MEG-fMRI study of the primary somatosensory cortex using cross-modal correspondence analysis. NeuroImage. 2004; 22:120-133. [PubMed: 15110002]

Shmuel A, Leopold DA. Neuronal correlates of spontaneous fluctuations in fMRI signals in monkey visual cortex: implications for functional connectivity at rest. Hum. Brain Mapp. 2008; 29:751761. [PubMed: 18465799]

Shrout PE, Fleiss JL. Intraclass correlations: uses in assessing rater reliability. Psychol. Bull. 1979; 86:420-428. [PubMed: 18839484]

Singh KD, Barnes GR, Hillebrand A, Forde EM, Williams AL. Task-related changes in cortical synchronization are spatially coincident with the hemodynamic response. NeuroImage. 2002; 16:103-114. [PubMed: 11969322]

Soros P, Sokoloff LG, Bose A, Mcintosh AR, Graham SJ, Stuss DT. Clustered functional MRI of overt speech production. NeuroImage. 2006; 32:376-387. [PubMed: 16631384]

Spironelli C, Angrilli A. Developmental aspects of language lateralization in delta, theta, alpha and beta EEG bands. Biol. Psychol. 2010; 85:258-267. [PubMed: 20659528]

Stippich C, Freitag P, Kassubek J, Soros P, Kamada K, Kober H, Scheffler K, Hopfengartner R, Bilecen D, Radu EW, Vieth JB. Motor, somatosensory and auditory cortex localization by fMRI and MEG. Neuroreport. 1998; 9:1953-1957. [PubMed: 9674573] 
Strother S, Lange N, Anderson J, Schaper K, Rehm K, Hansen LK, Rottenberg D. Activation pattern reproducibility: measuring the effects of group size and data analysis models. Hum. Brain Mapp. 1997; 5:312-316. [PubMed: 20408234]

Szaflarski JP, Holland SK, Schmithorst VJ, Byars AW. fMRI study of language lateralization in children and adults. Hum. Brain Mapp. 2006; 27:202-212. [PubMed: 16035047]

Szaflarski JP, Holland SK, Jacola LM, Lindsell C, Privitera MD, Szaflarski M. Comprehensive presurgical functional MRI language evaluation in adult patients with epilepsy. Epilepsy Behav. 2008; 12:74-83. [PubMed: 17964221]

Taylor MJ, Bayless SJ, Mills T, Pang EW. Recognising upright and inverted faces: MEG source localisation. Brain Res. 2011; 1381:167-174. [PubMed: 21238433]

Tuunanen PI, Kavec M, Jousmaki V, Usenius JP, Hari R, Salmelin R, Kauppinen RA. Comparison of BOLD fMRI and MEG characteristics to vibrotactile stimulation. NeuroImage. 2003; 19:17781786. [PubMed: 12948732]

Vannest J, Rasmussen J, Eaton KP, Patel K, Schmithorst V, Karunanayaka P, Plante E, Byars A, Holland S. FMRI activation in language areas correlates with verb generation performance in children. Neuropediatrics. 2010; 41:235-239. [PubMed: 21210340]

Vartiainen J, Liljestrom M, Koskinen M, Renvall H, Salmelin R. Functional magnetic resonance imaging blood oxygenation level-dependent signal and magnetoencephalography evoked responses yield different neural functionality in reading. J. Neurosci. 2011; 31:1048-1058. [PubMed: 21248130]

Vrba J, Robinson S. Linearly constrained minimum variance beamformers, synthetic aperture magnetometry, and MUSIC in MEG applications. Conference Record of the Thirty-Fourth Asilomar Conference on Signals, Systems and Computers. 2000; 1:313-317.

Wang Y, Xiang J, Kotecha R, Vannest J, Liu Y, Rose D, Schapiro M, Degrauw T. Spatial and frequency differences of neuromagnetic activities between the perception of open- and closedclass words. Brain Topogr. 2008; 21:75-85. [PubMed: 18679788]

Wang X, Xiang J, Wang Y, Pardos M, Meng L, Huo X, Korostenskaja M, Powers SW, Kabbouche MA, Hershey AD. Identification of abnormal neuromagnetic signatures in the motor cortex of adolescent migraine. Headache. 2010; 50:1005-1016. [PubMed: 20487034]

Wang Y, Xiang J, Vannest J, Holroyd T, Narmoneva D, Horn P, Liu Y, Rose D, Degrauw T, Holland $\mathrm{S}$. Neuromagnetic measures of word processing in bilinguals and monolinguals. Clin. Neurophysiol. 2011; 122:1706-1717. [PubMed: 21414839]

Wilke M, Schmithorst VJ, Holland SK. Assessment of spatial normalization of whole-brain magnetic resonance images in children. Hum. Brain Mapp. 2002; 17:48-60. [PubMed: 12203688]

Wilke M, Lidzba K, Krageloh-Mann I. Combined functional and causal connectivity analyses of language networks in children: a feasibility study. Brain Lang. 2009; 108:22-29. [PubMed: 18952275]

Williamson JM, Crawford SB, Lin HM. Resampling dependent concordance correlation coefficients. J. Biopharm. Stat. 2007; 17:685-696. [PubMed: 17613648]

Wise R, Hadar U, Howard D, Patterson K. Language activation studies with positron emission tomography. CIBA Found. Symp. 1991; 163:218-234. [PubMed: 1726146]

Xiang J, Wilson D, Otsubo H, Ishii R, Chuang S. Neuromagnetic spectral distribution of implicit processing of words. Neuroreport. 2001; 12:3923-3927. [PubMed: 11742212]

Xiang J, Wang Y, Chen Y, Liu Y, Kotecha R, Huo X, Rose DF, Fujiwara H, Hemasilpin N, Lee K, Mangano FT, Jones B, Degrauw T. Noninvasive localization of epileptogenic zones with ictal high-frequency neuromagnetic signals. J. Neurosurg. Pediatr. 2010; 5:113-122. [PubMed: 20043746]

Yuan W, Szaflarski JP, Schmithorst VJ, Schapiro M, Byars AW, Strawsburg RH, Holland SK. fMRI shows atypical language lateralization in pediatric epilepsy patients. Epilepsia. 2006; 47:593-600. [PubMed: 16529628]

Yuan W, Altaye M, Ret J, Schmithorst V, Byars AW, Plante E, Holland SK. Quantification of head motion in children during various fMRI language tasks. Hum. Brain Mapp. 2009; 30:1481-1489. [PubMed: 18636549] 
Zhang R, Wu T, Wang Y, Liu H, Zou Y, Liu W, Xiang J, Xiao C, Yang L, Fu Z. Interictal magnetoencephalographic findings related with surgical outcomes in lesional and nonlesional neocortical epilepsy. Seizure. 2011; 20:692-700. [PubMed: 21782477] 

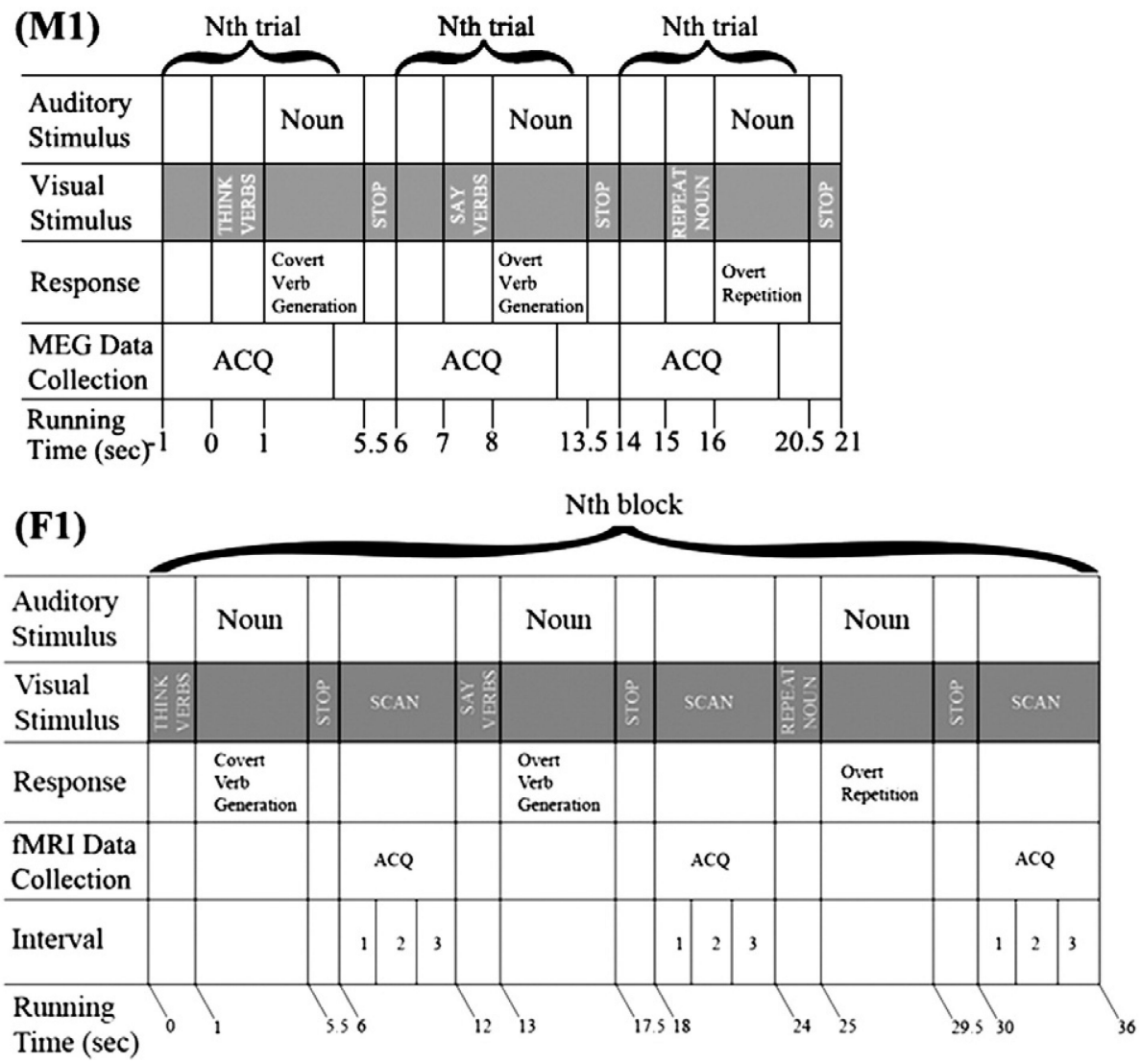

Fig. 1.

Timing diagram of the event-related verb-generation for MEG and fMRI task. MEG: 30 5-s Trials for each condition are recorded. FMRI: 1536 -s cycles for each phase of the paradigm are presented for a total scan time of 9 min. Overt verb generation performance is recorded for both MEG and fMRI sessions. 

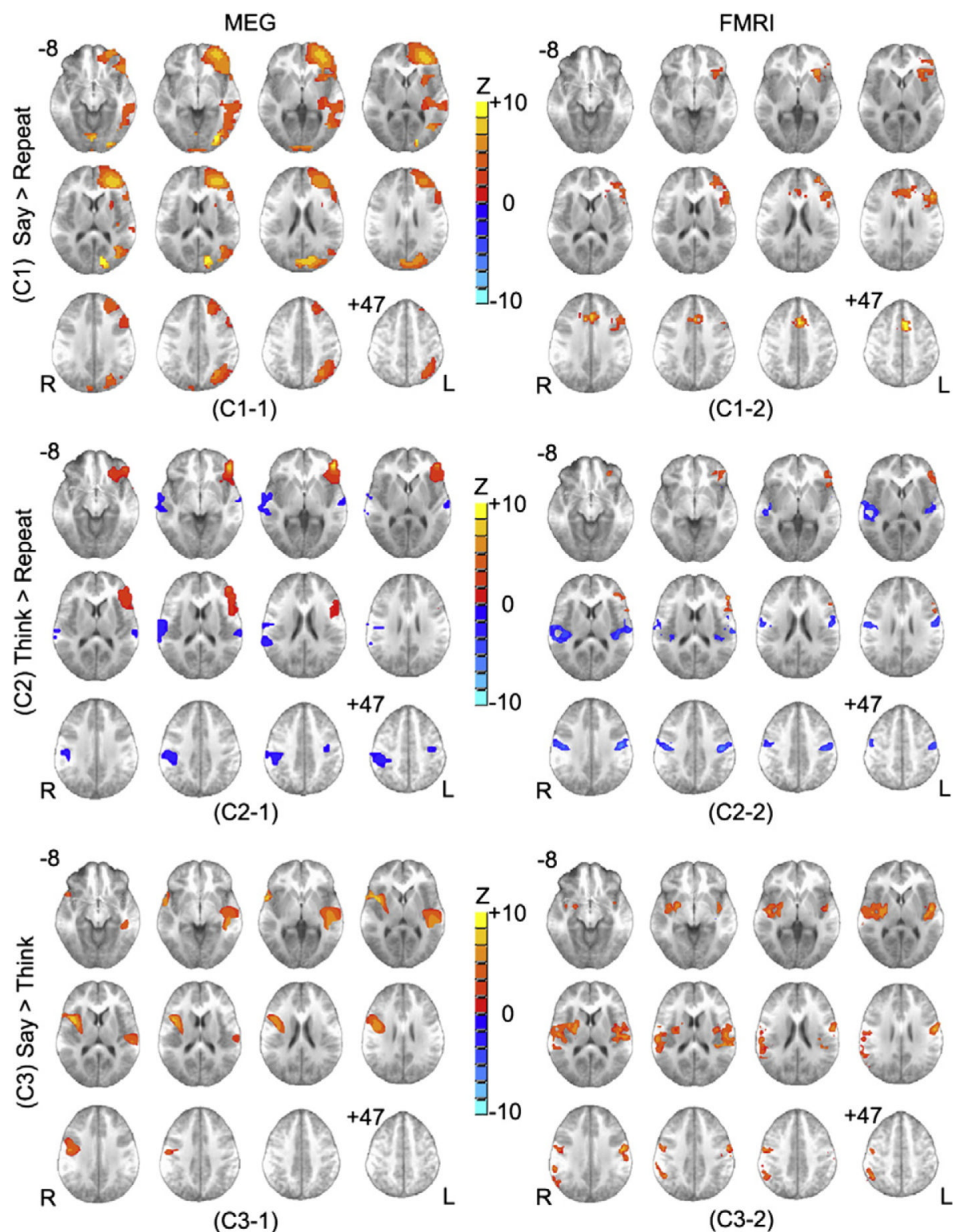

(C3-2)

Fig. 2.

Group composite activation maps of three contrasts from MEG (left) and fMRI (right) results (N=16): (C1) Say > Repeat (covert generation versus overt repetition); (C2) Think > Repeat (overt generation versus overt repetition); (C3) Say > Think (overt generation versus covert generation). Significant level for all contrasts: $\mathrm{p}<0.05$ corrected. Cluster size $>15$. Slice range: $\mathrm{Z}=-8$ to $+47 \mathrm{~mm}$ (Talairach coordinates) and $5 \mathrm{~mm}$ between each successive slice displayed. All images are in radiologic orientation. 


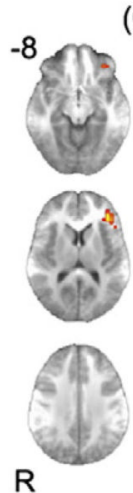

(C1) Say > Repeat
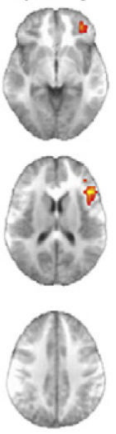

R
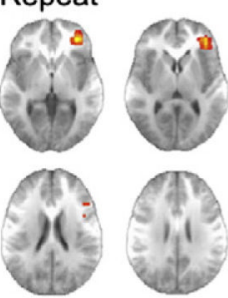

$+47$

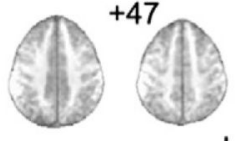

CCC Maps

(C2) Think > Repeat
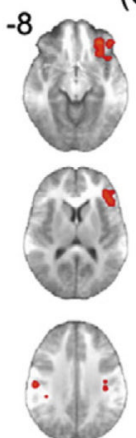

L R
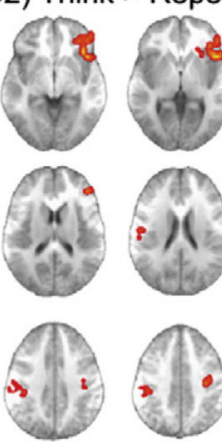

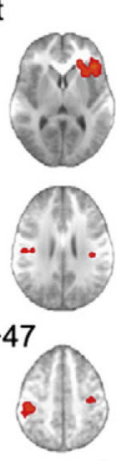

L R
(C3) Say > Think
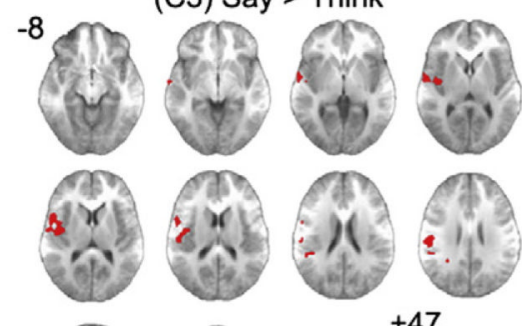

ح-
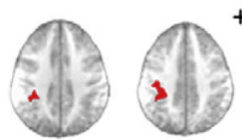

$+47$
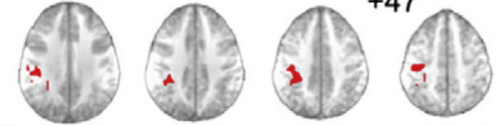

L

Fig. 3.

CCC maps of three contrasts. (C1) Say > Repeat (covert generation versus overt repetition); (C2) Think > Repeat (overt generation versus overt repetition); (C3) Say > Think (overt generation versus covert generation). Thresholded at 0.3 . Cluster size $>10$. Slice range: $Z=-$ 8 to $+47 \mathrm{~mm}$ (Talairach coordinates) and $5 \mathrm{~mm}$ between each successive slice displayed. All images are in radiologic orientation. 


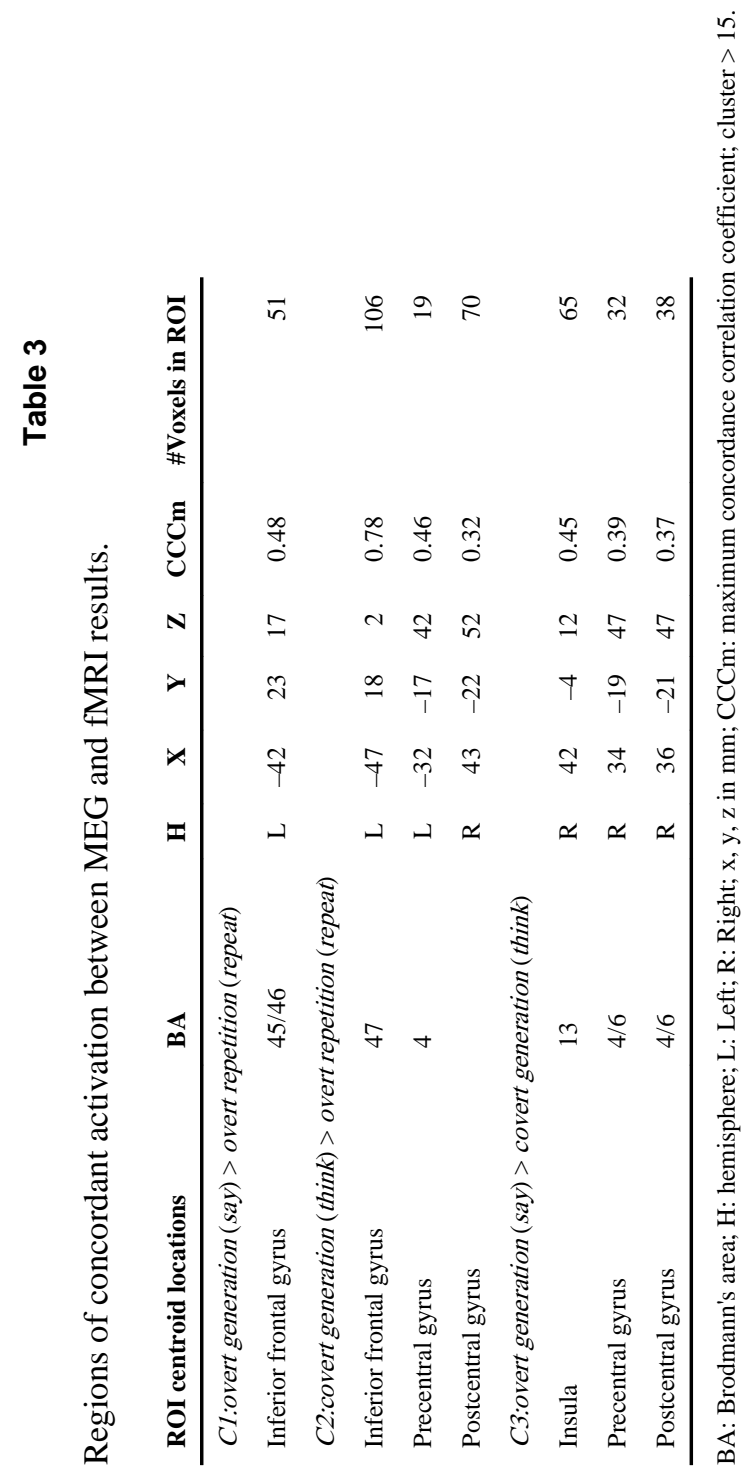

Brain Res. Author manuscript; available in PMC 2013 April 04. 